\title{
The Gorlin syndrome: a sporadic case
}

\section{Abdellah Dah Cherif, Younes Barbach, Mohammed Chouche, Sara Elloudi, Hanane Baybay, Fatima Zahra Mernissi}

Department of Dermatology, University Hospital Hassan II, Fez, Morocco

Corresponding author: Dr. Abdellah Dah Cherif, E-mail: abdellahdahcherif@gmail.com 


\title{
Le syndrome de Gorlin: Un cas sporadique
}

\section{Abdellah Dah Cherif, Younes Barbach, Mohammed Chouche, Sara Elloudi, Hanane Baybay, Fatima Zahra Mernissi}

\author{
Department of Dermatology, University Hospital Hassan II, Fez, Morocco
}

Corresponding author: Dr. Abdellah Dah Cherif, E-mail: abdellahdahcherif@gmail.com

Sir,

Le syndrome de Gorlin, ou le naevomatose basocellulaire (NBC), est une maladie héréditaire qui se caractérise par un ensemble d'anomalies du développement et par une prédisposition à développer de multiples carcinomes basocellulaires à un âge précoce, transmise sur le mode autosomique dominant [1]. On rapporte un cas sporadique de ce syndrome.

Une femme de 35 ans qui présente depuis 03 ans des multiples papules au niveau du visage, de petites tailles qui augmentent progressivement de tailles.

L' examen objectivait une tumeur ulcérobourgeonnante, croûteuse et hémorragique de $2 \mathrm{~cm}$ au niveau de l' aile du nez (Fig. 1). Un nodule avec des télangiectasies à centre ombiliqué faisant $1 \mathrm{~cm}$ sous l' œil gauche (Fig. 2), des nodules pigmentés au niveau des pommettes et deux nodules au niveau du dos du nez et un nodule érythémateux au niveau de la partie supèro-externe de la paupière supérieure de l' œil gauche, (Fig. 3) ainsi que des pits palmo-plantaires. La dermoscopie a objectivé des multiples nids ovoïdes avec une ulcération centrale, des vascularisation en tronc d' arbre et des télangiectasies à la périphérie des lésions. Les Radiographies standards des clavicules et du mandibule étaient normaux, avec a l' échographie pelvienne des multiples kystes ovariens. Lexamen histopathologique des lésions était en faveur de carcinome basocellulaire. La patiente a bénéficié d' un traitement chirurgical de toutes les lésions.

Le syndrome de Gorlin est une génodermatose rare. Il apparaît généralement dans le type à transmission autosomique dominante comme de multiples carcinomes basocellulaires [2]. Les Lésions solitaires

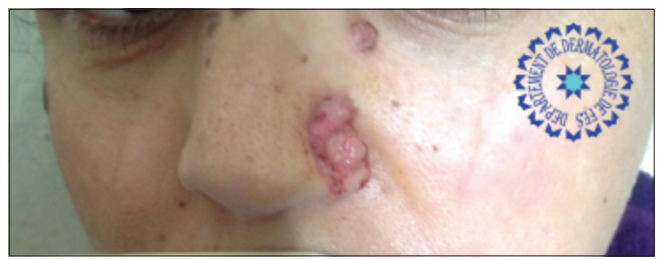

Figure 1: Tumeur ulcéro- bourgeonnante, croûteuse et hémorragique de $2 \mathrm{~cm}$ au niveau de l' aile du nez.

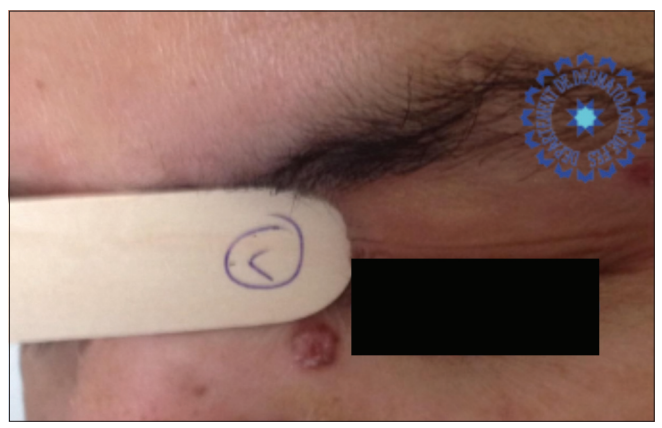

Figure 2: Un nodule avec des télangiectasies à centre ombiliqué faisant $1 \mathrm{~cm}$ sous l' œil gauche.

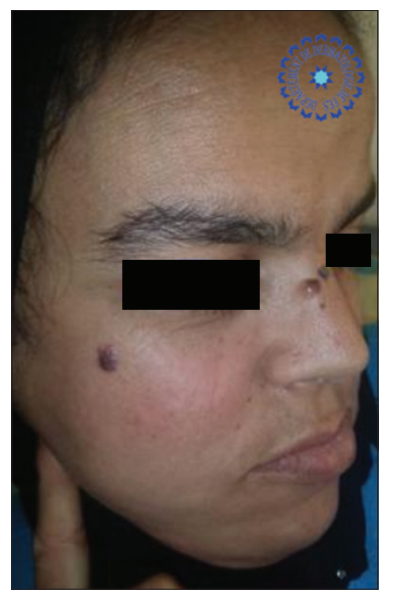

Figure 3: Des nodules pigmentés au niveau des pommettes et deux nodules au niveau du dos du nez et un nodule érythémateux au niveau de la partie supèro-externe de la paupière supérieure de l' œil gauche.

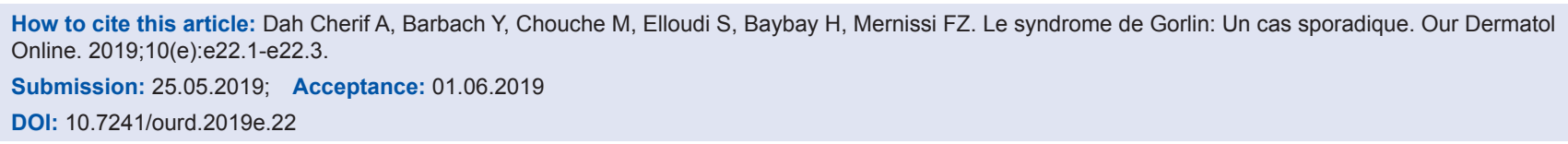


peuvent être excisées. Dans le cas de tumeurs multiples, la chirurgie peut être difficile. D'autres traitements ont été proposé dans la littérature (dermabrasion, électrochirurgie et la chirurgie au laser.) [3], dans notre cas on à opté pour exérèse chirurgical avec une bonne évolution.

Le syndrome de Gorlin est souvent transmis de manière dominante comme des multiples carcinomes basocellulaires d' ou la difficulté de réaliser l' exérèse de toute les lésions, nous rapportons un cas sporadique avec des multiples lésions traitées chirurgicalement.

\section{Consent}

The examination of the patient was conducted according to the Declaration of Helsinki principles.

\section{REFERENCES}

1. Lacombe D, Chateil JF, Fontan D, Battin J. Medulloblastoma in the nevoid basal cell carcinoma syndrome: case reports and review of the litterature. Genet Couns. 1990;1:273-7.

2. Hahn H, Wicking C, Zaphiropoulous PG, Gailani MR, Shanley S, Chidambaram A, et al. Mutations of the human homolog of drosophila patched in the nevoid basal cell carcinoma syndrome. Cell. 1996;85:841-51.

3. Pinatel B, Mojallal A. Prise en charge diagnostique et thérapeutique du carcinome cutané basocellulaire - Analyse des recommandations. Ann Chir Plast Esthét. 2012;57:92-105.

Copyright by Abdellah Dah Cherif, et al. This is an open-access article distributed under the terms of the Creative Commons Attribution License, which permits unrestricted use, distribution, and reproduction in any medium, provided the original author and source are credited.

Source of Support: Nil, Conflict of Interest: None declared. 